\author{
Katherine G. Akers \\ Department of Psychology \\ University of New Mexico \\ Albuquerque, NM 87131 \\ E-mail: kgakers@unm.edu
}

Derek A. Hamilton

Department of Psychology Department of Neurosciences University of New Mexico Albuquerque, NM 87131

\section{Comparison of Developmental Trajectories for Place and Cued Navigation in the Morris Water Task}

\begin{abstract}
Previous studies investigating the development of place and cued learning using the Morris water task are in disagreement regarding the day in development that each type of learning emerges. Here, place and cued navigation in the water task were examined in differently aged groups of young male and female rats $(P 17, P 18, P 19, P 20$, and $P 24)$ during a single day of training. When only distal cues were present, $P 20$ and $P 24$ but not younger rats learned the location of the hidden platform. In contrast, when a proximal cue marked the platform location, rats as young as P17 showed evidence of cue-controlled navigation, although only P18 and older rats exhibited cued learning. In line with most previous studies, these results indicate that cued learning emerges earlier in development than place learning and support a dissociation of developmental trajectories for the neural systems underlying the two types of navigation. (C) 2007 Wiley Periodicals, Inc. Dev Psychobiol 49: 553-564, 2007.
\end{abstract}

Keywords: rat; spatial learning; spatial navigation; episodic memory; learning strategy; water maze; hippocampus; striatum; caudate; sex difference; ontogeny

\section{INTRODUCTION}

Young rats display learning and memory function in a wide range of cognitive tasks remarkably early in development. In general, rats can perform relatively simple tasks such as delay conditioning very soon after the required sensory or motor systems become functional, whereas learning in more complex tasks such as trace conditioning or spatial tasks is not apparent until days or weeks later (Bachevalier \& Beauregard, 1993; Dumas, 2005; Stanton, 2000). One spatial task that can be configured to test different types of learning is the Morris water task (Morris, 1981). In a cued version of the task,

Received 9 January 2007; Accepted 5 March 2007

Correspondence to: K. G. Akers

Published online in Wiley InterScience

(www.interscience.wiley.com). DOI 10.1002/dev.20227 rats can escape a pool of water by navigating to a platform that is marked by a proximal cue; this task assesses the ability to learn an association between navigation to the proximal cue and escape from the water. In a place version of the task, only distal visual cues are present; this task assesses the ability to learn to escape from the water by navigating to the platform's fixed spatial location relative to a constellation of distal cues. The basic behavioral and neurobehavioral dissociations between cued and place navigation in the water task are well-established in adult animals (Morris, 1981; Morris, Garrud, Rawlins, \& O'Keefe, 1982; Redhead, Roberts, Good, \& Pearce, 1997; Sutherland, Kolb, \& Whishaw, 1982; Sutherland et al., 2001), however, whether these forms of learning and navigation can be dissociated in terms of their developmental trajectories has not been unequivocally established. One study reported that cued and place learning in the water task emerge on the same day of development (Brown \& Whishaw, 2000), although other studies report that rats exhibit cued learning at least a couple days earlier in development than place learning

(c) 2007 Wiley Periodicals, Inc. 
(Rudy \& Paylor, 1988; Rudy, Stadler-Morris, \& Albert, 1987; Schenk, 1985). The finding that cued learning developmentally precedes place learning supports the theory that cued and place learning rely on different neural systems (O'Keefe \& Nadel, 1978; White \& McDonald, 2002) and suggests a dissociation in the maturation of these systems.

A major debate within the previous literature on the development of spatial navigation in the rat concerns the precise point in development at which place learning emerges. Although several water task studies report that place learning does not emerge until between postnatal day (P) 19 and 21 (Brown \& Kraemer, 1997; Kraemer \& Randall, 1995; Rudy \& Paylor, 1988; Rudy et al., 1987; Tonkiss, Shultz, \& Galler, 1993), others assert that such ability emerges as early as P17 (Carman, Booze, \& Mactutus, 2002; Carman \& Mactutus, 2001). Such disparities in the age that place learning is first observed may be due to different methodologies utilized by these studies, including differences in the temporal distribution of training trials (Kraemer \& Randall, 1995), the size of the swimming pool (Carman \& Mactutus, 2001), whether body temperature was maintained throughout training (Brown \& Whishaw, 2000), and the saliency of the distal cue environment. One common feature of most previous developmental water task studies, however, is that they utilized a protocol consisting of multiple days of training, which renders it difficult to accurately determine the exact day on which place learning abilities emerge. Although one study used a less ambiguous approach of training separate groups of differently-aged rats within a single day, only a few ages of rats were examined (Brown \& Whishaw, 2000). Finally, many previous developmental studies have assessed performance in the water task using a limited number of behavioral measures, resulting in a less-than-complete characterization of both cued and place navigation in pre-weanling rats.

Here, we tested young male and female rats at five separate ages (P17, P18, P19, P20, and P24) in either place or cued versions of the Morris water task. All training and testing for each age group was given during a single day to allow for unambiguous identification of the first day in development at which place and cued learning emerge. Because hypothermia is known to impair performance in the water task (Iivnonen, Nurminen, Harri, Tanila, \& Puolivali, 2003; Moser \& Anderson, 1994; Rauch, Welch, \& Gallego, 1989), and young rats may be particularly susceptible to hypothermia due to their small size, care was taken to maintain body temperature throughout training and testing. Performance in the water task, particularly during the post-training probe trial, was assessed using several dependent measures to provide a detailed characterization of cued and place navigation for the ages of rats examined.

\section{METHODS}

\section{Animals}

All procedures were in accordance with the Institutional Animal Care and Use Committee at the University of New Mexico and conformed to the guide for the Care and Use of Laboratory Animals (National Research Council, 1996). Nine pregnant LongEvans hooded rats (Charles River Laboratories, Raleigh, NC) arrived at the department 10 days prior to giving birth. The day of birth was designated as P0. Litter sizes at birth ranged from 10 to 17 pups. Litters were culled to 10 pups within $24 \mathrm{hr}$ of birth, with equal numbers of male and female pups kept in each litter if possible. Pups were housed with their dam in plastic cages $(51 \mathrm{~cm} \times 25 \mathrm{~cm} \times 22 \mathrm{~cm})$ with a 12-hr light/dark cycle (lights on at 8:00 am). Food and water were provided in the home cages ad libitum. Pre-weanling rat pups $(n=80)$ underwent Morris water task testing on P17, P18, P19, P20, or P24. Based on previous research (Rudy et al., 1987; Schenk, 1985; Tonkiss et al., 1993), P24 rats were expected to display clear evidence of both place and cued learning, thus this age was included to provide an assessment of relatively good performance with which to compare the relatively poorer performance expected in the younger rats. On each day, eight rats were tested in the cued task and eight rats tested in the place task. In this manner, each rat was tested on only one day and in one task. Rats were assigned to cued and place tasks such that, on a given testing day, the rats in each task were comprised of equal or nearly equal numbers of males and females from unique litters. This resulted in each litter contributing a roughly equivalent number of males and females to the cued and place tasks across all testing days. At the end of water task testing, rats were ear-notched and returned to their home cage with dam and siblings.

\section{Apparatus}

The water task apparatus consisted of a white pool $1.5 \mathrm{~m}$ in diameter, $48 \mathrm{~cm}$ in height, and filled to a depth of $27 \mathrm{~cm}$ with $\sim 22^{\circ} \mathrm{C}$ water. The water was made opaque by the addition of a small amount of white non-toxic powdered paint. The platform $(15 \mathrm{~cm} \times 15 \mathrm{~cm})$ was made of white plastic and covered in a wire grid to assist rats in climbing onto it. The platform was located $\sim 1 \mathrm{~cm}$ below the surface of the water for both cued and place tasks. For the cued task, the platform location was marked by attaching a black plastic sphere $(9 \mathrm{~cm}$ in diameter, $11.5 \mathrm{~cm}$ in height) $11.5 \mathrm{~cm}$ above the center of the platform with a metal rod. Several distal visual cues were located around the testing room and remained constant for both cued and place tasks. Thus, in the cued task, both a proximal cue and distal cues were present, whereas in the place task, only distal cues were present. Behavior in the water task was videotaped by an overhead camera attached to a digital camcorder. Videos were transferred to a Linux workstation for tracking and analysis of swim paths.

\section{Morris Water Task}

Each rat was given six sessions of four trials within a single day of training. The platform remained in the center of the SW quadrant throughout training for both cued and place tasks. Rats were 
released from one of four locations that were equally spaced around the perimeter of the pool $(\mathrm{N}, \mathrm{S}, \mathrm{E}$, or W) with their heads facing the pool wall. For the first and third trials within a session, rats were released from a location close to the platform (S or W). For the second and fourth trials, rats were released from a location far from the platform ( $\mathrm{N}$ or $\mathrm{E}$ ). Each release location was used once within a session, and the sequence of release locations was randomized across sessions for each rat. To limit exposure to the cool water, trials lasted a maximum of $30 \mathrm{~s}$; if a rat did not find the platform within $30 \mathrm{~s}$, it was retrieved by the experimenter and placed on the platform. Rats remained on the platform briefly ( $\sim 5 \mathrm{~s}$ ) before being removed by the experimenter; this duration was selected to further limit exposure to the water and to reinforce navigation to the platform by quickly removing the rat from the water. Dependent measures during the training period were length of swim path and latency to reach the platform. In addition, the proportion of trials during which direct paths were taken to the platform was calculated for each rat; direct paths were characterized by rats' immediate departure from the pool wall, swimming in the general direction of the platform, and reaching the platform with relatively short latency and path length. If a rat did not reach the platform before the end of the trial, a latency of $30 \mathrm{~s}$ was recorded. The intertrial interval was $15 \mathrm{~s}$, during which rats were placed in a holding cage. During the intersession interval $(\sim 10$ $\min )$, rats remained in their individual cages within the testing room. Rats were dried and warmed after each session of trials following a method similar to that used by Brown and Whishaw (2000). Immediately after the last trial in each session, rats were towel-dried in front of a heater until their fur was mostly dry. Rats were then returned to their individual cages, which contained ample bedding and were located in close proximity to a heater. Before the next block of trials, rats' core body temperature was measured using a rectal thermometer (Physitemp Instruments, Clifton, NJ). Rats were not run in the next session of trials until their core body temperature was within $1^{\circ} \mathrm{C}$ of baseline body temperature $\left(37^{\circ} \mathrm{C}\right)$.

After the last session, rats were given a probe trial during which the platform was removed from the pool; rats were released from the $\mathrm{N}$ or $\mathrm{E}$ and allowed to swim for $30 \mathrm{~s}$. Dependent measures during the probe trial were length of swim path to reach the target zone (i.e., the former platform location), latency to reach the target zone, initial heading error, number of target zone crossings, percent of time spent in each of the four quadrants, and average proximity to target zone. Initial heading error was defined as the degree of deviation from a direct trajectory to the target zone measured at $\sim 37 \mathrm{~cm}$ from the release location. Average proximity to the target zone was calculated by sampling the distance between the rat and the platform (six times per second) and dividing the sum of these distances by the total number of observations; this measure thus provides a more precise assessment of the spatial distribution of a rat's swim path compared to other probe trial measures such as time spent in each quadrant (Gallagher, Burwell, \& Burchinal, 1993).

\section{Data Analysis}

Initial statistical analyses were performed using repeated measures or univariate ANOVA. Follow-up comparisons were performed using Tukey's post hoc tests.

\section{RESULTS}

\section{Training}

Performance in the water task during the training period was initially analyzed using omnibus repeated measures ANOVA with Task, Age, and Sex as between-subject factors and Session as a within-subject factor. We found a significant Task $\times$ Age $\times$ Session interaction $(F(20,300)$ $=1.99, p=.008)$, a significant Age $\times$ Session interaction $(F(20,300)=6.45, p<.001)$, and significant main effects of Task $(F(1,60)=35.89, p<.001)$, Age $(F(4$, $60)=24.49, p<.001)$ and Session $(F(5,300)=26.50$, $p<.001)$ on length of swim path. An identical pattern of significant effects was found for latency to reach the platform $($ Task $\times$ Age $\times$ Session: $F(20,300)=1.67, p=$ .037 ; Age $\times$ Session: $F(20,300)=5.05, p<.001)$; Task: $F(1,60)=33.90, \quad p<.001 ;$ Age: $F(4,60)=51.42$, $p<.001$; Session: $F(5,300)=25.13, p<.001)$. No effects involving Sex were significant, thus this factor was dropped from further training analyses. Due to the presence of significant Task $\times$ Age $\times$ Session interactions, subsequent analyses of training data were performed separately for place and cued tasks.

Representative swim paths for each age of rats from the last session of the place task are shown in Figure 1A. Throughout the training period, swim paths of P17, P18, and P19 rats were circuitous and characterized by a large degree of thigmotaxis. On average, P17, P18 and P19 rats found the platform on their own during $26 \pm 3 \%, 34 \pm 3 \%$, and $42 \pm 6 \%$ of the total number of trials (mean $\pm S E M$ ), respectively. Although $\mathrm{P} 20$ rats frequently exhibited circuitous swim paths, occasional direct paths to the hidden platform were also observed; P20 rats found the platform during $62 \pm 5 \%$ of the trials. After the first couple of sessions, $\mathrm{P} 24$ rats consistently took direct paths to the platform, finding the platform during $82 \pm 2 \%$ of the trials. Univariate ANOVA with Age as a between-subject factor was conducted on the proportion of trials during which rats navigated directly to the hidden platform from the release location. We found a significant main Age effect $(F(4,35)=23.80, p<.001)$. P24 rats exhibited a greater proportion of direct swim paths than all younger ages of rats $(p<.05)$. Furthermore, P20 rats exhibited a greater proportion of direct paths than $\mathrm{P} 17$ rats $(p<.05)$. Overall, this pattern of data suggests modest place navigation in $\mathrm{P} 20$ rats and good place navigation in P24 rats, whereas no evidence for place navigation was observed among younger rats.

These impressions were largely supported by analyses of path length and latency to reach the platform during the place task training period. Path length and latency data across place task sessions for each age are shown in Figure 1B and C. Repeated measures ANOVAs with Age 
A
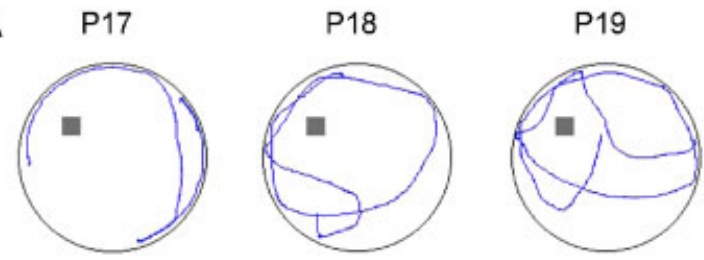

P24
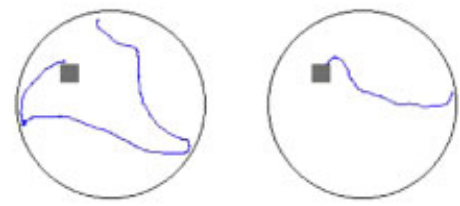

C

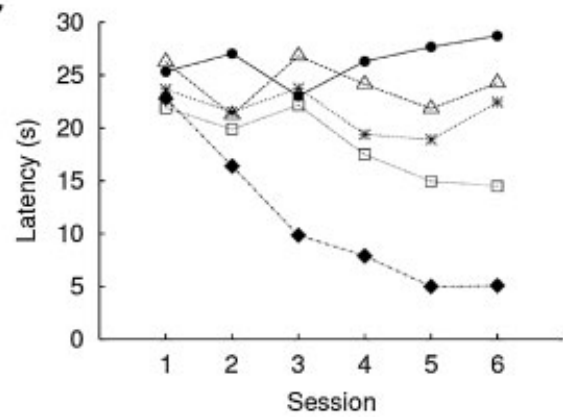

FIGURE 1 Place task training. (A) Representative swim paths for each age of rats during the last session. (B) Length of swim path across sessions. (C) Latency to reach platform across sessions. [Color figure can be viewed in the online issue, which is available at www.interscience.wiley.com.]

as a between-subject factor and Session as a withinsubject factor revealed a significant Age $\times$ Session interaction (path length: $F(20,175)=5.05, p<.001$; latency: $F(20,175)=4.08, p<.001)$ as well as significant main effects of Age (path length: $F(4,35)=8.92$, $p<.001$; latency: $F(4,35)=34.32, p<.001)$ and Session (path length: $F(5,175)=9.48, \quad p<.001 ;$ latency: $F(5,175)=8.34, p<.001)$ on both measures. These Age $\times$ Session interactions were further analyzed by performing separate repeated measures ANOVAs within each age group. P24 and P20 rats were found to exhibit improved performance across sessions of the place task, evidenced by significant decreasing linear trends for path length $(\mathrm{P} 20: F(1,7)=11.61, p=.011 ; \mathrm{P} 24: F(1,7)=$ $121.95, p<.001)$ and latency $(\mathrm{P} 20: F(1,7)=9.12$, $p=.019 ; \mathrm{P} 24: F(1,7)=104.80, p<.001) . \mathrm{P} 24$ rats also exhibited significant quadratic trends for both path length $(F(1,7)=10.48, p=.014)$ and latency $(F(1,7)=12.75$, $p=.009)$, with significant improvements in performance between sessions 1 and $3(p<.01)$ and no significant changes after session 4 -a pattern similar to that typically seen in adult rats. P17, P18, and P19 rats showed no significant improvement across sessions considering either path length or latency. When comparing differences among ages in place task performance during the last session of the training period, we found that P24 rats reached the hidden platform faster $(p<.05)$ and with shorter path lengths $(p<.05)$ compared to P20, P19, P18, and P17 rats. Furthermore, P20 rats reached the hidden platform faster $(p<.05)$ and with shorter path lengths $(p<.05)$ than $\mathrm{P} 19, \mathrm{P} 18$, and $\mathrm{P} 17$ rats, with the exception that P20 and P18 rats did not significantly differ with respect to path length.

Representative swim paths for each age from the last session of the cued task are shown in Figure 2A. Throughout the training period, $\mathrm{P} 17$ and $\mathrm{P} 18$ rats displayed highly circuitous and thigmotaxic swim paths. On average, $\mathrm{P} 17$ and $\mathrm{P} 18$ rats found the cued platform on their own during $48 \pm 7 \%$ and $40 \pm 6 \%$ of the total number of trials (mean $\pm S E M$ ), respectively; these escapes were characterized by an initial period of thigmotaxis followed by a direct path to the platform immediately after navigating away from the pool wall. Most of the paths taken by P19 and P20 rats were also circuitous and marked by thigmotaxis, although rats at these ages displayed a higher proportion of direct paths from the release location than younger rats; P19 and P20 rats found the platform during $73 \pm 6 \%$ and $71 \pm 5 \%$ of the trials, respectively. P24 rats consistently took direct paths to the cued platform, finding it during $93 \pm 3 \%$ of the trials. Univariate ANOVA with Age as a between-subject factor was conducted on the proportion of trials during which rats navigated directly to the cued platform from the release location. We found a significant main Age effect $(F(4,35)=17.83, p<.001) . \mathrm{P} 24$ rats exhibited a greater proportion of direct swim paths than all younger ages of rats $(p<.05)$. Furthermore, P20 rats exhibited a greater proportion of direct paths than P17 rats $(p<.05)$. Overall, this pattern of data suggests that the proximal cue controlled navigation to the platform for all ages of rats, 
A
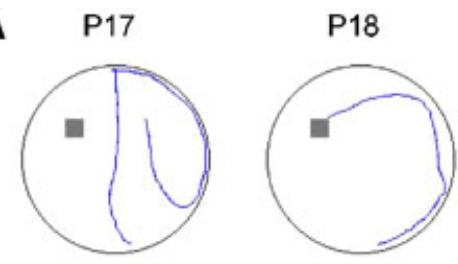

B

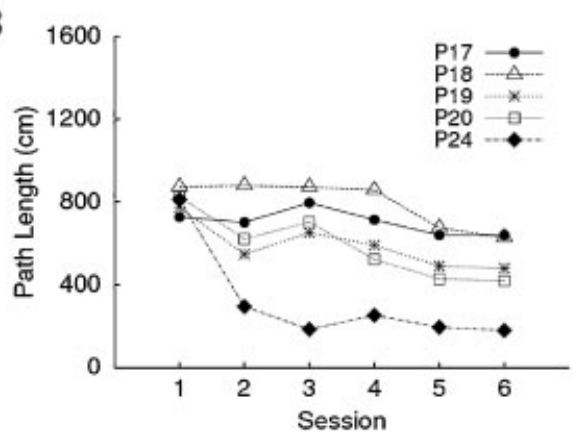

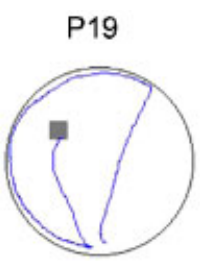

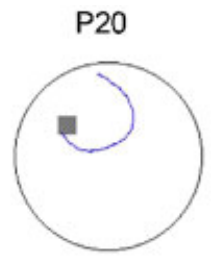

P24

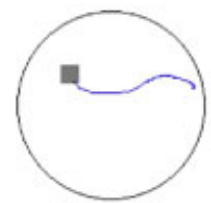

C

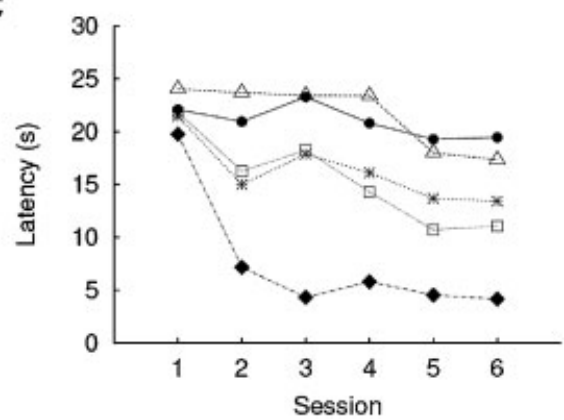

FIGURE 2 Cued task training. (A) Representative swim paths for each age of rats during the last session. (B) Length of swim path across sessions. (C) Latency to reach platform across sessions. [Color figure can be viewed in the online issue, which is available at www.interscience.wiley.com.]

but the quality of control by the cue improved with age: $\mathrm{P} 17$ and P18 rats displayed poor cued navigation, $\mathrm{P} 19$ and P20 rats displayed mediocre cued navigation, and P24 rats performed the cued task very well.

These impressions were partially supported by analyses of path length and latency to reach the platform during the cued task training period. Path length and latency data across cued task sessions for each age are shown in Figure 2B and $\mathrm{C}$. We found a significant Age $\times$ Session interaction (path length: $F(20,175)$ $=2.49, p=.001 ;$ latency: $F(20,175)=2.01, p=.009)$ as well as significant main effects of Age (path length: $F(1,35)=16.68, p<.001$; latency: $F(4,35)=19.90$, $p<.001)$ and Session (path length: $F(20,175)=17.42$, $p<.001$; latency: $F(20,175)=16.94, p<.001)$ on both measures. These Age $\times$ Session interactions were further analyzed by performing separate repeated measures ANOVAs within each age group. In contrast to the place task, in which only P20 and P24 rats showed improved performance across sessions, we found that $\mathrm{P} 18, \mathrm{P} 19, \mathrm{P} 20$, and P24 rats exhibited significant improvements in performance across sessions in the cued task, evidenced by decreasing linear trends for both path length (P18: $F(1$, $7)=10.05, p=.016 ; \mathrm{P} 19: F(1,7)=7.84, p=.027 ; \mathrm{P} 20$ : $F(1,7)=14.45, p=.007 ; \mathrm{P} 24: F(1,7)=59.19, p<.001)$ and latency $(\mathrm{P} 18: F(1,7)=14.10, p=.007$; P19: $F(1$, $7)=7.65, p=.028 ; \mathrm{P} 20: F(1,7)=15.57, p=.006 ; \mathrm{P} 24$ : $F(1,7)=65.22, \quad p<.001)$. P24 rats also exhibited significant quadratic trends for both measures (path length: $F(1,7)=23.55, p=.002$; latency: $F(1,7)=$
20.24, $p=.003$ ), with significant improvements in performance between sessions 1 and $2(p<.01)$ and no significant changes after session 3. P17 rats showed no significant improvement across sessions considering either path length or latency. When comparing differences among ages in cued task performance during the last session of the training period, we found that P24 rats reached the platform faster $(p<.05)$ and with shorter path lengths $(p<.05)$ than $\mathrm{P} 17, \mathrm{P} 18$, and $\mathrm{P} 19$ rats, with no further differences among age groups.

To determine whether the failure of P17 rats to exhibit improved performance across cued task sessions was due to an inability of rats at this age to see the proximal cue (rats' eyes opened around P15), we compared performance between P17 rats in the cued and place tasks. Univariate ANOVA with Task as a between-subject factor revealed a significant main effect of Task on path length $(F(1,14)=20.77, p<.001)$ and latency $(F(1,14)=$ $15.05, p=.002)$ during the last session of the training period, indicating that $\mathrm{P} 17$ rats performed better in the cued task than in the place task and providing evidence that P17 rats were able to utilize the visual cue to locate the platform.

\section{Probe Trial}

Performance in the water task during the probe trial was initially analyzed using omnibus univariate ANOVA with Task, Age, and Sex as between-subject factors. We found significant Task $\times$ Age interactions on heading error $(F(4$, 
$60)=2.71, p=.038)$, average proximity to the target zone $(F(4,60)=7.62, p<.001)$, and percent time spent in the target zone quadrant $(F(4,60)=2.73, p=.037)$. Significant main Age effects were found on target zone path length $(F(4,60)=10.29, p<.001)$, target zone latency $(F(4,60)=17.57, p<.001)$, number of target zone crossings $(F(4,60)=35.04, p<.001)$, average proximity to the target zone $(F(4,60)=28.94, p<.001)$, and percent time spent in the target zone quadrant $(F(4,60)=11.13$, $p<.001)$. Significant main Task effects were found on number of target zone crossings $(F(1,60)=11.61$, $p=.001)$ and percent time in target zone quadrant $(F(1$, $60)=4.29, p=.043$ ). Only one significant effect involving Sex was found on one dependent measure (main Sex effect on heading error: $F(1,60)=4.68, p=.035$; males $>$ females), thus this factor was dropped from further probe trial analyses. Due to the presence of significant Task $\times$ Age interactions on multiple probe trial measures, subsequent analyses of probe trial data were performed separately for place and cued tasks.

Representative swim paths for each age from the probe trial of the place task are shown in Figure 3A. For the most part, P17 rats never crossed the target zone during the probe trial, but rather exhibited highly circular and thigmotaxic swim paths similar to those observed during training. Most P18 and P19 rats crossed the target zone once during the probe trial, but this crossing was rarely the result of a direct path to the target zone; overall, swim paths of P18 and P19 rats remained circuitous and largely thigmotaxic. P20 rats displayed swim paths that were less thigmotaxic than those of younger rats; a large proportion of $\mathrm{P} 20$ rats crossed the target zone once or twice but did not display highly focused searching near the target zone. All P24 rats swam more or less directly to the target zone and persisted in swimming near the target zone throughout the probe trial. Together, these observations suggest that P24 rats had clearly learned the location of the hidden platform, whereas evidence for place learning in younger rats was minimal, particularly for rats younger than $\mathrm{P} 20$.

Dependent measures from the probe trial of the place task are shown in Figure 3B-G. Significant main Age effects were found for all probe trial measures (target zone path length: $F(4,35)=5.49, p=.002$; target zone latency: $F(4,35)=8.97, p<.001$; heading error: $F(4$, $35)=4.65, p=.004$; target zone crossings: $F(4,35)=$ $18.17, p<.001)$; average proximity: $F(4,35)=40.36$, $p<.001$; percent time in target zone quadrant: $F(4$, $35)=12.93, p<.001)$. P24 rats clearly displayed superior probe trial performance compared to younger rats-they reached the target zone faster than P19, P18, and P17 rats $(p<.05)$, exhibited shorter path lengths to the target zone and displayed less heading error than P18 and P17 rats $(p<.05)$, and crossed the target zone more often, swam with closer proximity to the target zone, and spent more time in the target zone quadrant than P20, P19, P18, and $\mathrm{P} 17$ rats $(p<.05)$. $\mathrm{P} 20$ rats displayed modestly better probe trial performance compared to younger rats-they reached the target zone faster and swam with closer proximity to the target zone compared to $\mathrm{P} 18$ and $\mathrm{P} 17$ rats $(p<.05)$. Finally, $\mathrm{P} 19$ rats were found to swim with closer proximity to the target zone during the probe trial compared to P18 and P17 rats $(p<.05)$. To determine whether rats displayed a preference for searching in the target zone quadrant compared to the other three quadrants during the probe trial, we performed repeated measures ANOVA with Age as a between-subject factor, Quadrant as a within-subject factor, and percent of time in each quadrant as a dependent measure. We found a significant Age $\times$ Quadrant interaction $(F(12$, $105)=4.39, p<.001)$ and a significant main Quadrant effect $(F(3,105)=3.89, p=.011)$. Separate repeated measures ANOVAs within each Age revealed a main Quadrant effect for P24 rats $(F(3,21)=21.78, p<.001)$; specifically, P24 rats spent the majority of the probe trial searching in the quadrant containing the target zone. A significant main Quadrant effect was also found for P17 rats $(F(3,21)=3.52, p=.033)$; these rats spent the majority of the probe trial searching in a quadrant adjacent to the one that contained the target zone.

Representative swim paths for each age from the probe trial of the cued task are shown in Figure 4A. P17, P18, $\mathrm{P} 19$, and P20 rats rarely crossed the target zone and, overall, displayed no tendency to search near the target zone during the probe trial. All P24 rats swam directly to the target zone after being released but, in contrast to P24 rats in the place task, did not display a highly focused search near the target zone throughout the remainder of the trial. This suggests that-at least among older ages of rats-the presence of a proximal cue during training may have overshadowed the distal cues, resulting in a disruption in performance when the cued platform was removed from the pool during the probe trial.

Dependent measures from the probe trial of the cued task are shown in Figure 4B-G. Significant main Age effects were found on nearly all probe trial measures (target zone path length: $F(4,35)=6.64, p<.001$; target zone latency: $F(4,35)=9.65, p<.001$; heading error: $p>.05$; target zone crossings: $F(4,35)=22.03, p<.001$; average proximity: $F(4,35)=6.93, p<.001$; percent time in target zone quadrant: $F(4,35)=3.39, p=.019)$. Similar to the P24 rats in the place task, P24 rats in the cued task in general displayed superior performance during the probe trial compared to younger rats-they reached the target zone faster, displayed shorter path lengths to the target zone, and crossed the target zone more often than $\mathrm{P} 20, \mathrm{P} 19, \mathrm{P} 18$, and $\mathrm{P} 17$ rats $(p<.05)$, swam with closer proximity to the target zone than P19 and P18 

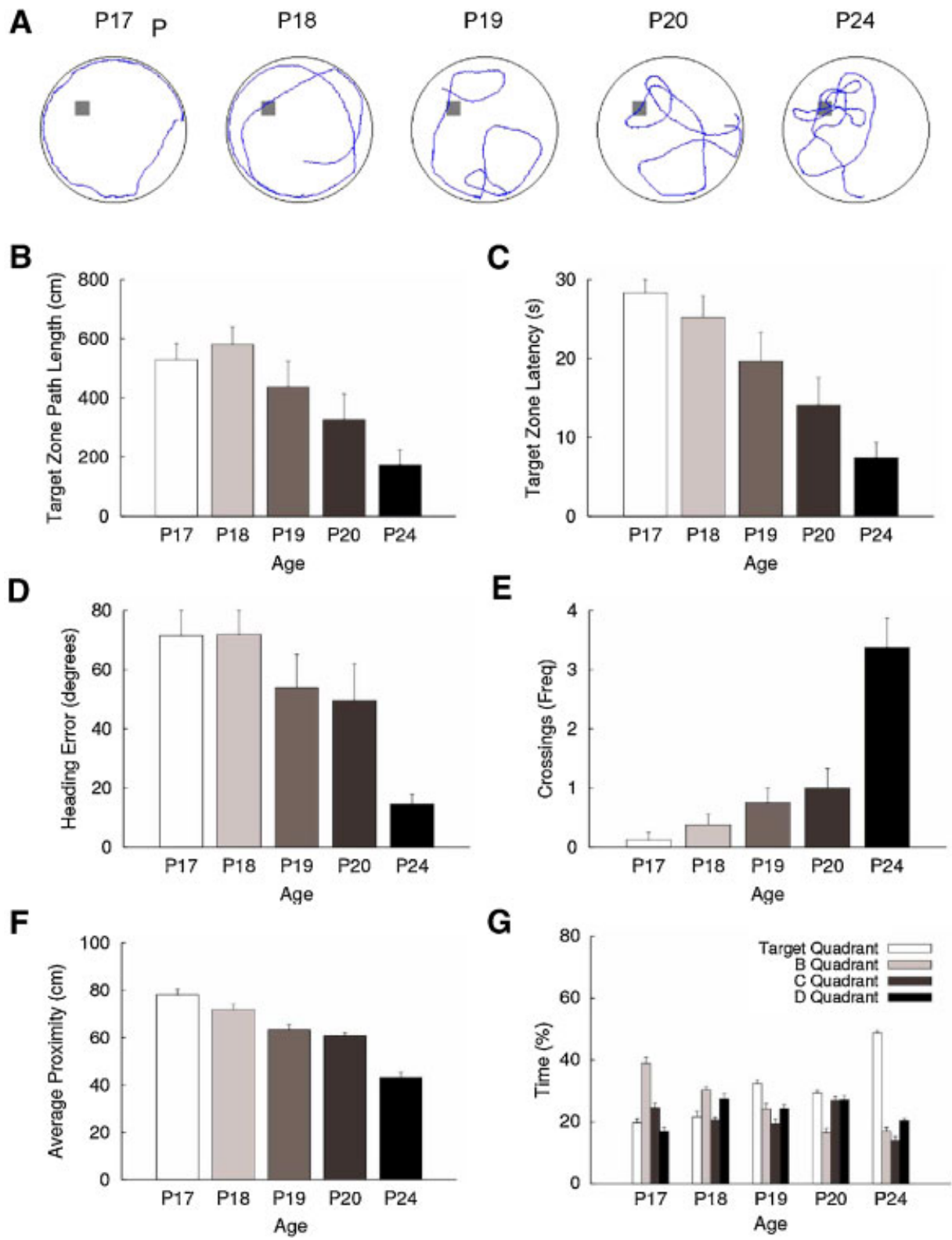

FIGURE 3 Place task probe trial. (A) Representative swim paths for each age. (B) Length of initial swim path to target zone. (C) Initial latency to reach target zone. (D) Degree of initial heading error to the target zone. (E) Frequency of target zone crossings. (F) Average proximity to target zone. (G) Percentage of time spent in each quadrant. [Color figure can be viewed in the online issue, which is available at www.interscience.wiley.com.]

rats $(p<.05)$, and spent more time in the target zone quadrant than $\mathrm{P} 18$ rats $(p<.05)$. No further significant differences were found among age groups. Repeated measures ANOVA with Age as a between-subject factor and Quadrant as a within-subject factor revealed a significant Age $\times$ Quadrant interaction on percent of time spent in the different quadrants $(F(12,105)=1.86$, $p=.048)$. Separate repeated measures ANOVAs within each Age revealed a main Quadrant effect only for P24 rats $(F(3,21)=5.37, p=.007)$; these rats spent large percentages of time searching in the target zone quadrant as well as in an adjacent quadrant.

To determine whether the proximal cue overshadowed the distal cues during training, probe trial performance was compared between same-aged rats trained in the place versus cued tasks. Univariate ANOVA with Task as a between-subject factor revealed that $\mathrm{P} 24$ rats in the place task displayed less heading error $(F(1,14)=9.03$, 
A

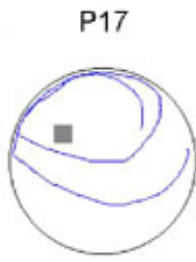

B

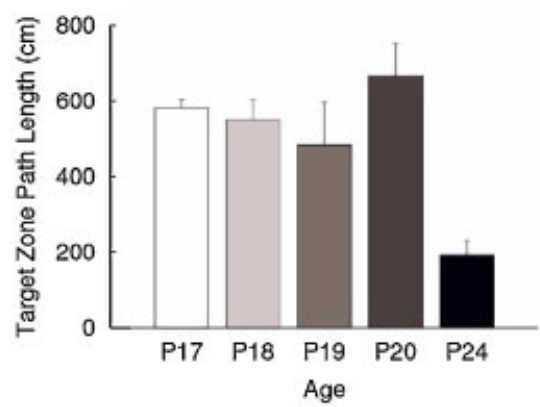

D

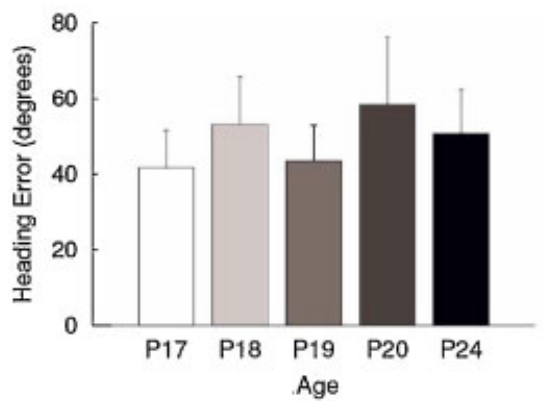

$\mathbf{F}$

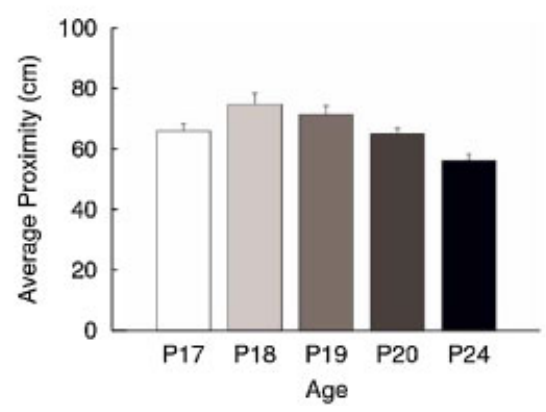

P19

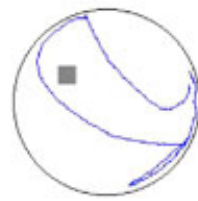

P24
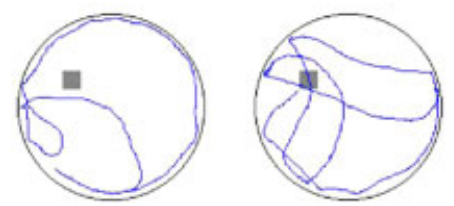

C

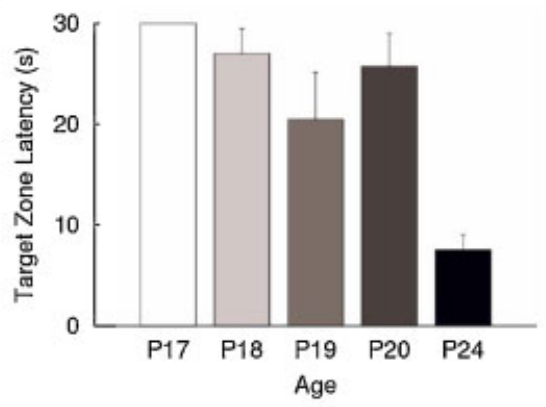

E

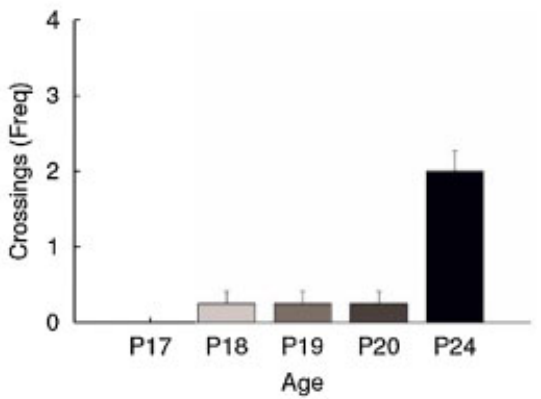

G

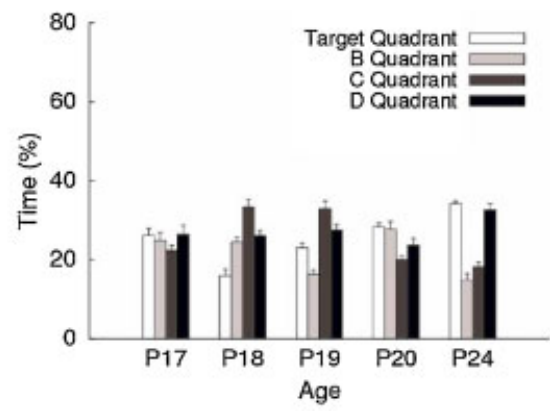

FIGURE 4 Cued task probe trial. (A) Representative swim paths for each age. (B) Length of initial swim path to target zone. (C) Initial latency to reach target zone. (D) Degree of initial heading error to the target zone. (E) Frequency of target zone crossings. (F) Average proximity to target zone. (G) Percentage of time spent in each quadrant. [Color figure can be viewed in the online issue, which is available at www.interscience.wiley.com.]

$p=.009)$, crossed the target zone more often $(F(1$, $14)=5.92, p=.029)$, swam with closer proximity to the target zone $(F(1,14)=19.23, p<.001)$, and spent a larger percent of time in the target zone quadrant $(F(1$, $14)=24.56, p<.001)$ compared to $\mathrm{P} 24$ rats in the cued task. Furthermore, P20 rats trained in the place task reached the target zone faster $(F(1,14)=5.94, p=.029)$ and with shorter path lengths $(F(1,14)=7.74, p=.015)$ compared to P20 rats trained in the cued task. These results are consistent with an overshadowing effect, which suggests that the relatively poorer probe trial performance among P20 and P24 rats in the cued versus place tasks may be explained by a greater reliance on a proximal cue over distal cues when a proximal cue is present during training. 


\section{DISCUSSION}

We assessed place and cued navigation in separate groups of differently aged male and female rats (P17, P18, P19, P20, and P24) during a single day of training in the Morris water task. In the place task, only distal room cues were available to guide navigation to a hidden platform in a fixed location, whereas in the cued task, a proximal cue marking the platform location was also present. In the place task, P20 and P24 rats but not younger rats showed improved performance across the training period. During a probe trial given at the end of the training period, P20 rats displayed modest evidence of learning the spatial location of the platform, whereas strong evidence of spatial learning was found for P24 rats. In the cued task, the proximal cue controlled navigation for all ages, although only P18 and older rats showed improved performance across the training period. No consistent difference in performance between male and female rats was observed. These results indicate that cued navigation emerges earlier in development than place navigation, suggesting a dissociation in the development of the neural systems that underlie the two types of navigation.

The point in development at which the ability to place navigate emerges has been a matter of debate in previous literature, with estimates ranging from 17 to 21 days of age (Brown \& Kraemer, 1997; Brown \& Whishaw, 2000; Carman et al., 2002; Carman \& Mactutus, 2001; Kraemer \& Randall, 1995; Rudy \& Paylor, 1988; Rudy et al., 1987; Tonkiss et al., 1993). Because nearly all these studies utilized protocols consisting of multiple, consecutive days of training, however, it is difficult to determine the precise day in development at which place navigation was first observed, particularly because the critical probe trial that assesses spatial learning is typically not administered until after the completion of training. Although some researchers (e.g. Carman et al., 2002; Carman \& Mactutus, 2001) tend to identify the day in development at which spatial navigation emerges in terms of rats' ages at the onset of training, a more conservative approach is to restrict judgment of the initial emergence of spatial learning to the final day of training and/or the day a probe trial is given. Moreover, an even less ambiguous approach for identifying the precise day in development at which place learning emerges is to administer all training and test trials within a single day, as was done in the present study as well as one previous study (Brown \& Whishaw, 2000), although this approach may entail certain disadvantages such as fatigue, hypothermia, or a retardation in acquisition rate that occurs with massed compared to spaced training (Commins, Cunningham, Harvey, \& Walsh, 2003; Kraemer \& Randall, 1995; but see Spreng, Rossier, \& Schenk, 2002 for exception).
When considering the results of previous water task studies in terms of rats' ages at the completion of training rather than the onset of training, the available data, including those from the present study, provide fairly consistent evidence regarding the point in development at which place navigation emerges. In experiments performed by Rudy and colleagues (1987), P20 and P21 rats that began training on $\mathrm{P} 18$ and $\mathrm{P} 20$, respectively, displayed persistence in searching at the former platform location during a post-training probe trial; P21 rats also displayed decreased latencies to locate the hidden platform across the last day of training. This same laboratory also found that P21 rats learned the spatial location of a platform after receiving 2 or 3 days of training in a cued task (Rudy \& Paylor, 1988). Brown and Kraemer (1997) and Tonkiss et al. (1993) trained rats from P20 to P21; in both studies, P21 rats displayed decreased latencies to find the hidden platform across training days and persistence in searching at the former platform location during a probe trial. In the present study, place task training occurred during a single day; P20 rats were the youngest group to exhibit a decrease in latency and path length to the platform across the training period and modest persistence in searching at the former platform location during the probe trial. Although these five studies concur that place navigation emerges around P20 or P21, others have reported place navigation in rats at a slightly younger age. In a study by Kraemer and Randall (1995), training commenced on P17 or P18 and ended on P19; regardless of the day that training began, P19 rats displayed decreased latencies to reach the hidden platform across training days as well as persistence in searching at the former platform location during a probe trial. Mactutus and colleagues $(2002,2001)$ trained rats from P17 to P19 and found that P19 rats displayed modest persistence at the former platform location during a probe trial, although within-day decreases in latencies during training were not observed. Finally, Brown and Whishaw (2000) reported that P19 rats displayed decreased latencies across a single day of training and persistence in searching at the former platform location during a post-training probe trial. Taken together, all previous water task studies as well as the present study are in agreement that place learning in the rat emerges within a fairly well-defined period during development - between 19 and 21 days of age.

Although our finding of initial place learning in P20 rats is in the range of that found in previous studies, it is somewhat curious that we did not observe place learning slightly earlier in development (i.e. P19) as has been reported by a number of previous studies (Brown \& Whishaw, 2000; Carman et al., 2002; Carman \& Mactutus, 2001; Kraemer \& Randall, 1995). One reason for this discrepancy may be the difference between present and previous studies in the duration of training 
trials. In an effort to prevent hypothermia and fatigue in the young rats, we used trials lasting a maximum of $30 \mathrm{~s}$, which is shorter than the 60-90 s trial duration used in previous developmental studies. This shorter trial duration may have resulted in a reduced number of trials during which rats found the hidden platform without experimenter assistance, which, in turn, may have impeded rats' learning of how to navigate to the platform on their own. Because we administered a large number of training trials, however, the total number of trials during which our P19 rats found the platform on their own is likely comparable to that in previous developmental studies. For instance, our P19 rats found the hidden platform during 10 trials on average (out of a possible 24) - a number that is probably similar to the total number of trials during which P19 rats found the platform in Brown and Whishaw's (2000) and Kraemer and Randall's (1995) studies, which utilized 12 and 16 training trials, respectively. Thus, it is unlikely that our P19 rats did not learn to place navigate because of an insufficient number of trials that were reinforced without experimenter intervention. Another possibility is that the shorter trial duration used in the present study may have served to lessen rats' persistence in searching near the hidden platform location. That is, during training, rats that might have narrowly missed finding the platform early in a trial may have been disallowed the opportunity to return to the platform location on their own due to a short trial duration. Thus, longer trial durations may ultimately enhance measures of persistence - a possibility that may, at least in part, account for the failure of P19 rats in the present study to persist in searching at the former platform location during the probe trial. Apart from trial duration, other methodological differences between the present and previous studies that may have influenced performance of P19 rats in the place task include the temporal distribution of training trials (Kraemer \& Randall, 1995), the size of the pool (Carman \& Mactutus, 2001), or the saliency of the distal cue environment.

Of the previous water task studies that investigated the development of cued navigation in relation to place navigation, all demonstrated that rats can cue navigate earlier in development than they can place navigate. Rudy and colleagues $(1988,1987)$ reported that P17 rats exhibited shorter latencies to reach the platform when it was marked by a proximal cue than when only distal cues were present, and both P16 and P17 rats displayed decreased latencies to find the cued platform across a single day of training. Brown and Whishaw (2000) found that P18 rats-the youngest age included in their studyreached the platform faster in a cued task than in a place task. Furthermore, in the present study, P17 rats-the youngest age examined-that were trained in the cued task reached the platform faster than same-aged rats trained in the place task, although they did not take direct paths to the cued platform or exhibit decreases in latency to reach the platform across training. Because these four studies found no evidence of place navigation until rats were 19-21 days of age, they demonstrate that rats are capable of utilizing a proximal cue to guide navigation earlier in development than they are able to navigate using only distal cues. This developmental dissociation in the emergence of cued and place navigation in young rats is consistent with that observed in human children, in which the ability to place navigate is not typically observed until several years after cued navigation is possible (Leplow et al., 2003; Overman, Pate, Moore, \& Peuster, 1996). Such a developmental dissociation of cued and place navigation supports the theory that the two types of navigation are controlled by separate neural systems (O'Keefe \& Nadel, 1978; White \& McDonald, 2002) and suggests that these neural systems develop at different rates. Specifically, the initial emergence of cued navigation is believed to arise from the relatively early development of striatal function, whereas the later emergence of place navigation is thought to depend on delayed maturation of hippocampal function (Bachevalier \& Beauregard, 1993; Dumas, 2005; Stanton, 2000).

One issue that has often been overlooked in previous developmental water task studies is a possible effect of hypothermia on task performance. In adult mice, core body temperature drops $\sim 5-6^{\circ} \mathrm{C}$ over the course of five trials in quick succession, and this drop in temperature correlates with a reduction in swimming speed (Iivnonen et al., 2003). In adult rats, artificially lowering body temperature (Rauch et al., 1989) or brain temperature (Moser \& Anderson, 1994) to $\sim 7-9^{\circ} \mathrm{C}$ below baseline impairs acquisition and retention of the platform location and causes motor disturbances such as decreased swimming speed and difficulty in climbing onto the platform. Because of young rats' small size, they are likely to experience a severe drop in body temperature as a result of exposure to cool water, and such hypothermia may cause performance deficits that could mask learning ability in the water task. Thus, it may be imperative in developmental water task studies to prevent hypothermia in young rats-as was done in the present study-in order to accurately reveal the developmental emergence of place and cued navigation. It should be noted, however, that prevention of hypothermia is probably not a factor that could explain the discrepancy between the present study and Brown and Whishaw's (2000) study in terms of the day of development on which spatial learning was first observed, as both studies used similar techniques to maintain rats' body temperatures during training.

Although the existing water task studies are generally in accord concerning the developmental emergence of place navigation as well as the developmental dissociation 
between place and cued navigation, some issues are still in need of clarification. One such issue is a distinction between the control of an animal's navigation by a proximal cue and that animal's ability to learn about a relationship between a proximal cue and a goal location. For instance, in the present study, P17 rats' navigation was clearly under the control of the proximal cue, as rats of this age exhibited shorter latencies and path lengths to reach the platform in the cued task than in the place task. P17 rats, however, displayed no evidence of learning to escape the water by navigating directly to the cued platform; rather, evidence of cued learning was not observed until P18. Similarly, data from Brown and Whishaw (2000) indicate that P18 rats were capable of using a proximal cue to guide navigation, but decreasing latencies to reach the cued platform across sessions were not observed until P19. Because place navigation was also first observed on P19 in Brown and Whishaw's study, the authors concluded that cued and place learning exhibit a parallel developmental trajectory. Thus, the distinction between control of navigation by a proximal cue and the ability to associate a proximal cue with a goal location may complicate the identification of when cued navigation emerges relative to place navigation. Another difficulty in the existing literature, including the present study, is that in cued tasks, distal room cues are often present in the testing environment in addition to the proximal cue. As a consequence, it becomes impossible to distinguish whether animals are learning about the relationship of the platform location to the proximal cue, to the distal cues, or to both. This is particularly the case when cued learning and place learning are found to emerge on the same day of development, as in the case of Brown and Whishaw's study.

To address such issues in the current literature, perhaps different water task paradigms could be employed in future developmental studies. A paradigm that might be particularly useful could be derived from that introduced by Redhead et al. (1997) using adult rats. Specifically, young rats could undergo testing in either a place or a cued version of the water task; both tasks would utilize two proximal cues inside the pool, with a hidden platform consistently located under only one cue. In the place task, the two proximal cues would be visually identical and remain in fixed positions relative to the distal room cues; escaping the water in this task could be achieved only by learning to navigate toward the cue that is located in a specific place relative to the distal cues. In the cued task, the two proximal cues would be visually dissimilar and change locations from trial to trial; escaping the water in this task could be achieved only by learning to navigate toward the cue that is unique in terms of shape, color, and/ or pattern. Given that navigation of very young rats can clearly be controlled by proximal cues within the pool, such an experimental paradigm, although likely requiring multiple days of training, may reveal evidence of place learning in even younger animals than has been previously reported. Furthermore, these methods may provide a more sensitive test for dissociating cued and place navigation.

\section{NOTES}

We acknowledge Katrina Barnes, Henrik Gemoll, Sylwia Gutierrez, Mathew Sanchez, Andrea Watts, and Brandon Wesenberg for their assistance in data collection and analysis.

\section{REFERENCES}

Bachevalier, J., \& Beauregard, M. (1993). Maturation of medial temporal lobe memory functions in rodents, monkeys, and humans. Hippocampus, 3, 191-202.

Brown, R. W., \& Kraemer, P. J. (1997). Ontogenic differences in retention of spatial learning tested with the Morris water task. Dev Psychobiol, 30, 329-341.

Brown, R. W., \& Whishaw, I. Q. (2000). Similarities in the development of place and cue navigation by rats in a swimming pool. Dev Psychobiol, 37, 238-245.

Carman, H. M., Booze, R. M., \& Mactutus, C. F. (2002). Longterm retention of spatial navigation by preweanling rats. Dev Psychobiol, 40, 68-77.

Carman, H. M., \& Mactutus, C. F. (2001). Ontogeny of spatial navigation in rats: A role for response requirements? Behav Neurosci, 115, 870-879.

Commins, S., Cunningham, L., Harvey, D., \& Walsh, D. (2003). Massed but not spaced training impairs spatial memory. Behav Brain Res, 139, 215-223.

Dumas, T. C. (2005). Late postnatal maturation of excitatory synaptic transmission permits adult-like expression of hippocampal-dependent behaviors. Hippocampus, 15, 562578.

Gallagher, M., Burwell, R., \& Burchinal, M. (1993). Severity of spatial learning impairment in aging: Development of a learning index for performance in the Morris water maze. Behav Neurosci, 107, 618-626.

Iivnonen, H., Nurminen, L., Harri, M., Tanila, H., \& Puolivali, J. (2003). Hypothermia in mice tested in Morris water maze. Behav Brain Res, 141, 207-213.

Kraemer, P. J., \& Randall, C. K. (1995). Spatial learning in preweanling rats trained in a Morris water maze. Psychobiology, 23, 144-152.

Leplow, B., Lehnung, M., Pohl, J., Herzog, A., Ferstl, R., \& Mehdorn, M. (2003). Navigational place learning in children and young adults as assessed with a standardized locomotor search task. Br J Psychol, 94, 299-317.

Morris, R. G. M. (1981). Spatial localization does not require the presence of local cues. Learn Motiv, 12, 239260.

Morris, R. G. M., Garrud, P., Rawlins, J., \& O’Keefe, J. (1982). Place navigation is impaired in rats with hippocampal lesions. Nature, 297, 681-683. 
Moser, E. I., \& Anderson, P. (1994). Conserved spatial learning in cooled rats in spite of slowing of dentate field potentials. J Neuroscience, 14, 4458-4466.

O'Keefe, J., \& Nadel, L. (1978). The hippocampus as a cognitive map. Oxford: Oxford University Press/Clarendon Press.

Overman, W. H., Pate, B. J., Moore, K., \& Peuster, A. (1996). Ontogeny of place learning in children as measured in the radial arm maze, Morris search task, and open field task. Behav Neurosci, 110, 1205-1228.

Rauch, T. M., Welch, D. I., \& Gallego, L. (1989). Hypothermia impairs performance in the Morris water maze. Physiol Behav, 46, 315-320.

Redhead, E. S., Roberts, A., Good, M., \& Pearce, J. M. (1997). Interaction between piloting and beacon homing by rats in a swimming pool. J Exp Psychol: Anim Behav Proc, 23, 340350.

Rudy, J. W., \& Paylor, R. (1988). Reducing the temporal demands of the Morris place-learning task fails to ameliorate the place-learning impairment of preweanling rats. Psychobiology, 16, 152-156.

Rudy, J. W., Stadler-Morris, S., \& Albert, P. (1987). Ontogeny of spatial navigational behaviors in the rat: Dissociation of "proximal"- and "distal"-cue-based behaviors. Behav Neurosci, 101, 62-73.

Schenk, F. (1985). Development of place navigation in rats from weaning to puberty. Behav Neural Biol, 43, 69-85.

Spreng, M., Rossier, J., \& Schenk, F. (2002). Spaced training facilitates long-term retention of place navigation in adult but not in adolescent rats. Behav Brain Res, 128, 103-108.

Stanton, M. E. (2000). Multiple memory systems, development and conditioning. Behav Brain Res, 110, 25-37.

Sutherland, R. J., Kolb, B., \& Whishaw, I. Q. (1982). Spatial mapping: Definitive disruption by hippocampal or medial frontal cortical damage in the rat. Neurosci Lett, 31, 271-276.

Sutherland, R. J., Weisend, M. P., Mumby, D., Astur, R. S., Hanlon, F. M., \& Koerner, A, et al. (2001). Retrograde amnesia after hippocampal damage: recent vs. remote memories in two tasks. Hippocampus, 11, 27-42.

Tonkiss, J., Shultz, P., \& Galler, J. R. (1993). Long-Evans and Sprague-Dawley rats differ in their spatial navigation performance during ontogeny and at maturity. Dev Psychobiol, 25, 567-579.

White, N. M., \& McDonald, R. J. (2002). Multiple parallel memory systems in the brain of the rat. Neurobiol Learn Mem, 77, 125-184. 